overestimate children's skills in identifying safe and dangerous traffic situations.

Methods We selected 289 pupils from three primary schools in Styria covering urban and rural traffic areas. These children were subdivided into four age groups spanning one year each. The test series consisted of 4 different samples (a traffic mat, a simulation, photos and live action video) with 10 recognition tasks for each (5 safe, 5 unsafe traffic situations) and varying points of view (e. g. from an outside observer, a bird's eye view and from a child's point of view). Over two days, each test was carried out by briefed students and with standardised face-to-face interviews of approx. 20 minutes.

Results Only $75 \%$ of the questions were answered correctly, correlating with a clearly defined age trend: the younger the children, the more likely they were to base their assessment of the degree of danger on one single factor: the presence or absence of a vehicle on the road. The percentage of correct answers was higher in older children but all age groups had the most difficulty interpreting the sample from their own points of view. It was easier for children to identify the dangers from an outsider's perspective. In addition, the best performance was shown when using live-action videos.

Conclusions The younger the children, the more difficult it is for them to identify dangerous situations on the road. From the age of 10 onward they are able to interpret most traffic situations correctly, but fail $20 \%$ of the time. Hence parents should be informed of the benefit to accompanying their children in the first few weeks of each primary school year and advised not to overestimate their child's traffic-assessing skills.

For traffic safety education purposes it is essential to develop materials consisting of live-action videos and showing the same scenery from different points of view. That way, children will be able to look beyond an obstacle, to think ahead and increase their overall traffic safety.

\section{CONTINUOUS APPROACH TO ROAD SAFETY EDUCATION}

Natalia Agre. Non-Governmental Organisation "Road Safety Russia", Russia

\subsection{6/injuryprev-2016-042156.544}

Background Road safety culture takes years to form; that is why it is necessary to start road safety education from the very first years of life. More importantly, it is only possible to reach its full potential with the provision of a continuous educational system enforced by the combination of infrastructural, legislative and law enforcement measures.

Objective To provide continuous road safety education with the help of outreach and educational activities.

To develop programs accompanying road users from their early years to adulthood: trainings for parents in perinatal centres; interactive programs for kindergarteners and primary school students; educational classes for middle and high school students; highlighting the importance of road safety for driving school students; reminding adults of their responsibility towards the safety of children.

To bring road safety to the top of the priority list concerning knowledge and skills of vital importance taught to children from an early age.
Results Road Safety Russia campaigns combining interventions effective for various target audiences (children, adults, parents, drivers, passengers) has resulted in a steady decrease in road accident rates in Russia. A significant contribution was made to the fact of drivers and passengers buckling up, drivers sticking to the speed limits, children and their parents being more enthusiastic when it comes to road safety initiatives, constantly improving statistics on child restraint systems use.

Conclusions Continuous approach to education is crucial in the context of providing knowledge on road safety: a child that has always been seated in a child car seat, always used helmets when riding a bike, always crossed the road with their mother via a crosswalk will not risk their life and disregard road safety in their adulthood.

\section{STRENGTHENING COMMUNITY ACTION TO REDUCE MOTORCYCLE ACCIDENTS IN DEZFUL, IRAN}

${ }^{1}$ Nastaran Keshavarz Moahmmadi, ${ }^{2}$ Maryam Mazaheri. ${ }^{1}$ Shaid Behesti University of Medical Sciences, Iran; ${ }^{2}$ Dezful University of Medical Sciences, Iran

\subsection{6/injuryprev-2016-042156.545}

Background Iran is among countries with highest road traffic injuries and mortality. Road traffic mortality is the second cause of death of Iranian population. The share of motorcycles in road injuries and mortalities varies in different cities, but it is highest (60 percent) in a southern city of Iran, Dezful.

Methods An action research project which is partly reported here was designed to strengthen community action to reduce road traffic accidents. Through many advocacy measures, a team including more than 25 public and private stakeholders was formed. By holding several meetings and also conducting complementary individual interviews and comprehensive content analysis, current situation, local determinists of the problem and most highly effective change strategies were determined. A 6 months educational campaign was developed. A valid developed questionnaire was used to measure individual determinates of unsafe motorcycle driving. Pretest and post test was conducted to evaluate the effect of campaign on the rate of motorcycle related accidents and level of change in individual determinants of unsafe driving.

Results Early analysis of formative research showed that absence of safety culture, hot and humid climate of the city and also narrow structure of the streets were among most important determinants of unsafe behaviour. Process evaluation showed that for the first time, such collaboration and intersectoral action has been formed at city level with effective participation of the community. The result of outcome evaluation, the rate of change in road traffic accidents and individual determinants would be reported in this presentation after final data analysis.

Conclusions Effectiveness and sustainability of success of any road safety intervention depends on deep and comprehensive understanding of local determinates of the problem, local solutions and assets and also the quality of community participation in the program. 


\section{Alcohol, Drugs and Medicines Related Injuries}

\section{Post Mon 1.13}

\section{COUGH MIXTURES ABUSE AMONGST ADOLESCENT STUDENTS IN DONGGUAN, CHINA}

Zuhui Chen, Junfang Xian, Shangyun Sun, Haixia Mo, Haiyan Xia, Jinrong Lin, Haohui Yu. First Affiliated Hospital of Jinan University, 613 West Huangpu Avenue, Guangzhou, China

\subsection{6/injuryprev-2016-042156.546}

Background Cough mixtures can help or harm. Very few literatures involved cough mixtures abuse (abbreviation to CMA) while we often have clinical cases basically of adolescent abusers. This research aims to the prevalence of CMA among adolescent students in Dongguan, China.

Methods Analyses were based on a cross-sectional survey of students (N 8 529) from all 22 middle and high schools in Dongguan, China. Logistic regression models were computed to examine prospective risk factors of CMA behaviour. Cough mixtures were defined in those prescription medicine drinks containing codeine component for the upper respiratory tract treatment purpose originally.

Results The very prevalence of CMA varied from 5.36\% to $12.54 \%$ in all 8 age groups (12 to 19 years old) during the past one year before investigation. Male students were more vulnerable with a rate of $8.17 \%$ in comparison to female $5.93 \%$ ( 3.263 , p 0.017). Prospective risk factors of CMA included single parent family (OR 3.047, 95\% CI: 2.184 to 3.946), depression or anxiety (OR 2.851, 95\% CI: 1.014 to 3.415 ), high study stress (OR 1.954, 95\% CI: 1.245 to 3.214 ), and convenience to obtain (OR 1.856, 95\% CI: 1.012 to 2.547). Drug dependence was the main adverse consequence of CMA from acute symptoms like palpitation (7.48\%), illusion (5.26\%), and coma (2.58\%) to chronical changes such as weak appetite and mal-digestion $(85.47 \%)$, difficulty to sleep $(80.12 \%)$, and decline of memories (78.24\%).

Conclusions CMA endangering adolescent students calls for multidisciplinary enforcement and education intervention in Dongguan, China.

\section{ACCIDENTS AMONG SUBSTANCE USE DEPENDENT PATIENT SEEKING TREATMENT: COCAINE VERSUS OTHER SUBSTANCES}

${ }^{1} \mathrm{FJ}$ Alvarez, ${ }^{2,3} \mathrm{~L}$ Rodriguez-Cintas, ${ }^{2,3} \mathrm{C}$ Roncero, ${ }^{2} \mathrm{C}$ Daigre, ${ }^{1} \mathrm{MC}$ del Río, ${ }^{2} \mathrm{CA}$ Abad, ${ }^{2} \mathrm{~N}$ Vela, ${ }^{2} \mathrm{~A}$ Herrero-Fernandez, ${ }^{2,3} \mathrm{~L}$ Grau-López, ${ }^{2,3}{ }^{3}$ Ros-Cucurrull, ${ }^{2,3,4} \mathrm{M}$ Casas. ${ }^{1}$ Pharmacology, Faculty of Medicine, University of Valladolid, Spain; 'Vall d'Hebron University Hospital, Addiction and Dual Diagnosis Unit. Psychiatry, Barcelona, Spain; ${ }^{3}$ Universidad Autonoma De Barcelona, Psychiatry, Barcelona, Spain; ${ }^{4}$ Vall d'Hebron University Hospital, Psychiatry, Barcelona, Spain

\subsection{6/injuryprev-2016-042156.547}

Background Cocaine use has been associated with traffic road accident involvement. Early studies showed frequent accident involvement of patients in treatment for Substance Use Disorders. The aim of the study is to analyse the history of accidents and risk behaviours in cocaine dependent patients (CD) and other substance type dependent patients (OD).

Methods 182 patients in treatment for their Substance Use Disorders from January 2014 to September 2015 were included in the study. History of accident involvement of any kind and risk behaviours was recorded, as well as follow up to 1 year. SPSS (23) was used for statistical analysis.

Results $45.2 \%$ of patients were CD and $54.8 \%$ were OD $(35.6 \%$ alcohol, $8.3 \%$, cannabis, $5.8 \%$ opioid and $5.1 \%$ other drugs). $30.3 \%$ were women and $69.7 \%$ were men, aged 43.7 (mean) years $(\mathrm{SD}=13) .65 .6 \%$ were currently driving. Lifetime involvement in accidents was frequently reported by Substance Use Disorder patients (34.6\%), more likely among CD (40.4\%) than among OD (29.0\%). Furthermore, CD patients referred further potentially dangerous activities, such as driving under the influence of drugs $(65.9 \%$ vs. $33.3 \%)$ and driving with abstinence symptomatology $(41.7 \%$ vs $12 \%)$, more frequently than OD patients.

Conclusions Substance Use Disorder patients frequently drive and were frequently involved in accidents. Accident involvement and risk behaviours were more likely among cocaine dependent patients than those dependent on other drugs.

Supported by grants from: Plan Nacional sobre Drogas 2013 I044 and the Instituto de Salud Carlos III, Redes Temáticas de Investigación Cooperativa, Red de Trastornos Adictivos RD12/0028/00212

\section{ROADSIDE TESTING OF OPIATES IN ORAL FLUID OF SPANISH DRIVERS}

F Javier Álvarez, Inmaculada Fierro. Faculty of Medicine, University of Valladolid, Spain

\subsection{6/injuryprev-2016-042156.548}

Background Opiates could impair psychomotor performance, and driving under the influence of opiates is associated with increased accident risk. The aim of the study was: i) to determine the prevalence of opiates in Spanish drivers; and ii) to explore opiate drug usage (heroin, morphine, codeine and methadone), alone or in combination with other drugs.

Methods The 2008/9 DRUID database regarding Spain was used, including information on 3302 drivers. All drivers included in the study provided a saliva sample and mass-chromatographic analysis was provided in all cases. To determine prevalence, the sample was weighted according to traffic intensity and, for positive cases, DRUID project cutoffs were applied. To explore opiate use the sample was used unweighted and the detection of each substance was considered a positive result.

Results The prevalence of opiates in Spanish drivers was $0.5 \%$ (95\% CI: $=[0.3-0.8])$. Opiates, either alone or together with other drugs, were detected in 64 drivers. The average age $( \pm S D)$ was $35.53 \pm 11.72$ years and the majority of the cases were men (79.7\%). No case of morphine as medicinal use was observed. The specific metabolite of heroin 6-acetyl morphine (6-AM) was detected in the saliva of 34 drivers: in 8 cases alone, in 7 cases with methadone, multiple substance use being the rule. Codeine was detected in 26 drivers (in 10 drivers alone) and methadone in 24 drivers (in 10 drivers alone). Polydrug use was common in opiate user drivers (56.25\%). The most frequent non opiate drugs were cocaine $(\mathrm{n}=32)$, THC $(\mathrm{n}=14)$ and benzodiazepines $(\mathrm{n}=7)$.

Conclusions Driving with the presence of opiates is relatively infrequent. Polydrug use is common among Spanish drivers who are opiate users. Only $40 \%$ of methadone positive cases (likely on methadone maintenance programs) were using this substance alone. 